\title{
Access to Maternal Healthcare in Rural Bangladesh: A literature-based Analysis on Factors Affecting Maternal Healthcare Utilization in Bangladesh
}

\author{
Noor $\mathrm{R}^{1}$, Saif $A \mathrm{R}^{2}$ \\ DOI: https://doi.org/10.3329/jafmc.v16i1.53848
}

\begin{abstract}
In the last decade, Bangladesh has made substantial improvements of maternal and newborn mortality, such the maternal mortality ratio (MMR) had dropped from 574 per thousand live births in 1990 to 170 per thousand live births in 2013. Despite these improvements, Bangladesh is still lagging in achieving equity in service utilization. Literature manifested significant healthcare inequities across different socio-demographic factors and religious and cultural views. The primary aims of this analysis are -i) to assess the disparities in the quality and availability of maternal healthcare in Bangladesh and its relation to the service utilization in rural Bangladesh, ii) to identify demand-side interventions implemented in developing countries and relevant considerations to promote maternal healthcare utilization in rural Bangladesh. A narrative literature review was conducted using Rudolph Matas Library, Tulane University online resources, Google Scholar and PUBMED. Articles published between January 1st, 2015 and December 31st, 2018 describing quality, availability and utilization of maternal healthcare services; existing disparities and barriers in healthcare utilization in rural Bangladesh are included in the analysis. This analysis also includes articles describing the impact of demand-side interventions on healthcare utilization in developing countries. A total of 10 articles met the selection criteria. Bibliographies of selected articles were also searched to include additional information in this analysis. Reviews of the literature revealed a significant healthcare equity gap according to place of residences, wealth status, age at birth, and parity of mothers in Bangladesh. According to available study findings, the level of mothers' education, cultural and social views, women's employment status, distance of health facility, and perceived quality of care act as significant factors influencing maternal healthcare utilization in rural Bangladesh. Studies have shown that demand-side interventions considering cultural context and other existing barriers result in improved uptake of maternal healthcare and improved health outcome. Only availability of services cannot translate into better outcomes, unless those services are of acceptable quality and the population irrespective of their socio-demographic status can attain those services without any perceived barrier. The analysis suggested, along with ensuring availability and quality of healthcare, the need for demand-side interventions addressing existing barriers make services accessible to the rural communities in Bangladesh.
\end{abstract}

Key-words: Maternal healthcare in Bangladesh, Disparities in Utilization, Barriers to access care, Improved Health outcome.

\section{Introduction}

Health inequity is the foreground of development issues for most developing countries. In 2000, Millennium Development Goals (MDG) were developed and adopted by most countries around the globe to meet the needs of the world's poorest population and promote international development. The MDG era came to an end in 2015, and later, United Nations and United Nations member states adopted Sustainable Development Goals (SDGs) with a target to accomplish the goals by 2030 . The SDGs are an attempt to follow up on the successful creation of a united direction shaped by the Millennium Development Goals (MDGs) ${ }^{1}$. The third SDG deals with health, places emphasizes on universal health coverage (UHC) ${ }^{1}$ and implicitly indicates the need to establish an equitable distribution of health services and outcomes. However, literature reveals that approximately 290,000 women die every year due to pregnancy and childbirth, and $99 \%$ of maternal mortality occurs in the developing world ${ }^{2}$. Every day approximately 800 women die from preventable causes related to pregnancy and childbirth ${ }^{3}$ and the majority of maternal deaths occur in low and low-middle-income countries because of unavailable, inaccessible, unaffordable or poor-quality maternal health services ${ }^{4}$.

Bangladesh is one of those developing countries with high maternal mortality ratio (MMR), standing at 170 per thousand live births ${ }^{5}$. Although it has become a lower- middle income country with economic growth and achieved various targets of millennium development goals, the target on reduction of maternal mortality and utilization related to obstetric and reproductive services provided by skilled health personnel remains unmet ${ }^{2}$. There is persistent inequity in quality, availability, and uptake of health services in Bangladesh. Several factors influence this inequity across the nation, such as: wealth status (rich vs. poor), area of living (urban vs. rural), different religious views, social stigma and so on. Review of literature suggested targeted interventions are necessary, which will address the existing barriers for mothers to access the services available. Simultaneously, policy attention should be devoted to developing health systems that can offer quality care to meet the increased demand for services ${ }^{4}$.

\section{Rationale}

Over the last two decades, Bangladesh has made commendable progress in achieving a few maternal health-related MDG targets. For instance, MMR had dropped from 574 per thousand live births

1. Dr Rafat Noor, MBBS, MPH, MSc, Graduate Assistant, University of Alberta, Canada (E-mail: rafat1@ualberta.ca) 2. Maj Aumeo Rushdi Saif, MBBS, General Staff Officer-2 (Coord), Armed Forces Medical College (AFMC), Dhaka. 
in 1990 to 170 per thousand live births in 2013. In spite of this noticeable progress, Bangladesh is still lagging in achieving equity in service utilization. A common feature of the inequity is that the use of maternal health services has persistently been lower amongst the poorer and less educated, as well as those living in rural and remote areas. Women face several barriers to seek or reach accessible, high-quality care, such as lack of awareness regarding healthcare, lack of knowledge about available services, decision making autonomy and inability to pay for services, religious barrier, and social stigma. To improve healthcare utilization, as well as to ensure effective positive outcome achieved from the utilization, it is critical to understand the status of disparities and the existing barriers to access the healthcare in rural Bangladesh. Besides, an analysis including culturally sensitive demand-side interventions implemented in low-and middle-income countries is a high priority to determine the effective demand-side interventions in the context of Bangladesh.

\section{Research Hypothesis}

Along with improving the quality and availability of healthcare, implementation of culturally sensitive demand-side interventions is necessary to increase the utilization of maternal healthcare services in rural Bangladesh.

\section{Objectives}

To assess the disparities in the quality and availability of maternal healthcare in Bangladesh and its relation to service utilization in rural Bangladesh and to identify demand-side interventions implemented in developing countries and relevant considerations to promote maternal healthcare utilization in nations with strong cultural sensitivities.

\section{Materials and Methods}

A systemic literature search was conducted from 15 February 2015 to 31 May 2019 to find studies on maternal healthcare utilization in Bangladesh. This literature search was conducted using Rudolph Matas Library Tulane University online resources, Google Scholar and Pubmed. Key search terms that were used by the combination of words and phrases including: Availability of maternal healthcare in Bangladesh and Disparities in Utilization, Demand-side interventions and Maternal healthcare utilization in rural Bangladesh, availability of maternal healthcare in Rural Bangladesh and barriers to access care, Availability of maternal healthcare and Quality of Care in Rural Bangladesh, Demand-side interventions in lower and middle-income countries and maternal healthcare utilization, Demand-side interventions in lower and middle-income countries and Improved health outcome. Titles identified from selected search terms were reviewed to meet the criterion of being published between January 1st, 2015 and December 31st, 2018. For inclusion in the analysis, the articles are needed to describe, 1) quality and availability of maternal healthcare services in rural Bangladesh, 2) existing disparities in maternal healthcare service utilization in Bangladesh, 3) effects of barriers to access maternal healthcare on its uptake in rural Bangladesh and 4) impact of demand-side interventions on healthcare utilization in developing countries. Apart from the literature identified through this search, information added from other literature is included in the reference section. The focus of this search was to analyze quality, availability of maternal healthcare services and relevant factors (socio-economic, demand-side interventions) affecting utilization of maternal healthcare in Bangladesh.

\section{Results}

A total of 160 titles were identified and reviewed from the database of PubMed based on search terms. After reviewing the titles, abstract, background and discussion, 10 of the 160 articles were selected for analysis. The Figure-1 describes how the articles were utilized to reach the hypothesis.

1. Quality and availability of maternal healthcare services in rural Bangladesh: A report from Ministry of Health and Family Welfare published in collaboration with WHO, World Bank, Partnership for Maternal, Newborn and Child Health and Alliance for Health Policy and System Research ${ }^{6}$ in 2015 states that Bangladesh was on track for meeting the national MDG maternal mortality target of $143 / 1,00,000$ live births in 2013. This report ${ }^{6}$ also states that the rate of declining MMR averaged $5.5 \%$ per year which was slightly higher than the $5.4 \%$ rate required for achieving MDG ${ }^{5}$. This reduction in maternal mortality is attributed to the increased availability of maternal health services in the last decade. According to Pulok et al, between the years of 2004 and 2011, the coverage level of maternal health service increased for both antennal care (ANC) and facility birth ${ }^{4}$. In addition, to improve Primary Healthcare (PHC) service delivery, the Government of Bangladesh established 18,000 community clinics (CCs) across the country, including in hard reaching areas ${ }^{7}$. The main purpose of CCs is to provide essential care packages for women and children ${ }^{8}$. Studies reported that, these CCs are convenient for the rural women from the perspective of location. However, Bangladesh is still facing challenges to ensure quality in the healthcare facilities ${ }^{4,7,8}$. Lack of qualified health worker is one of the major causes behind this quality issue of healthcare in rural Bangladesh ${ }^{6}$. Moreover, due to other supply-side barriers including lack of infrastructure, unavailability of essential medicine, long waiting time and low perceived respects by rural mothers relate to lower quality of healthcare in rural areas of Bangladesh ${ }^{4,9}$.

2. Existing disparities in maternal healthcare service utilization in Bangladesh: Available research demonstrates that there are notable disparities in the utilization of maternal healthcare services in Bangladesh. The disparities were identified among richest and poorest households, urban and rural areas, across geographical divisions, also among other significant socio-demographic indicators including employment status, age and education. 


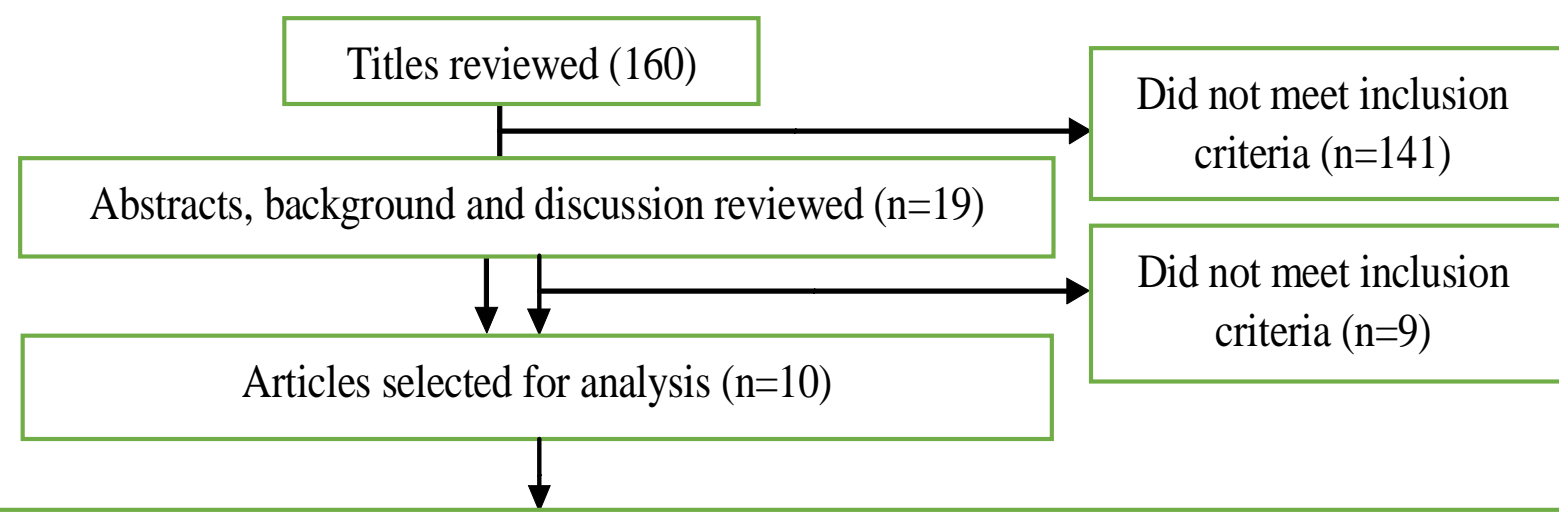

-Disparities in quality, availability and utilization of maternal healthcare services in Bangladesh

- Existing barriers to access the available services in rural Bangladesh

-Demand-side interventions to increase utilization of maternal healthcare services in low and middleincome countries

-Lack of demand-side interventions to address existing barriers to access services

-High-quality services are not available

-Women are not aware about their health needs and available services

- Enough services are not available to meet the created demand

- Implemented interventions are not culturally competent

- Improper implementation of interventions
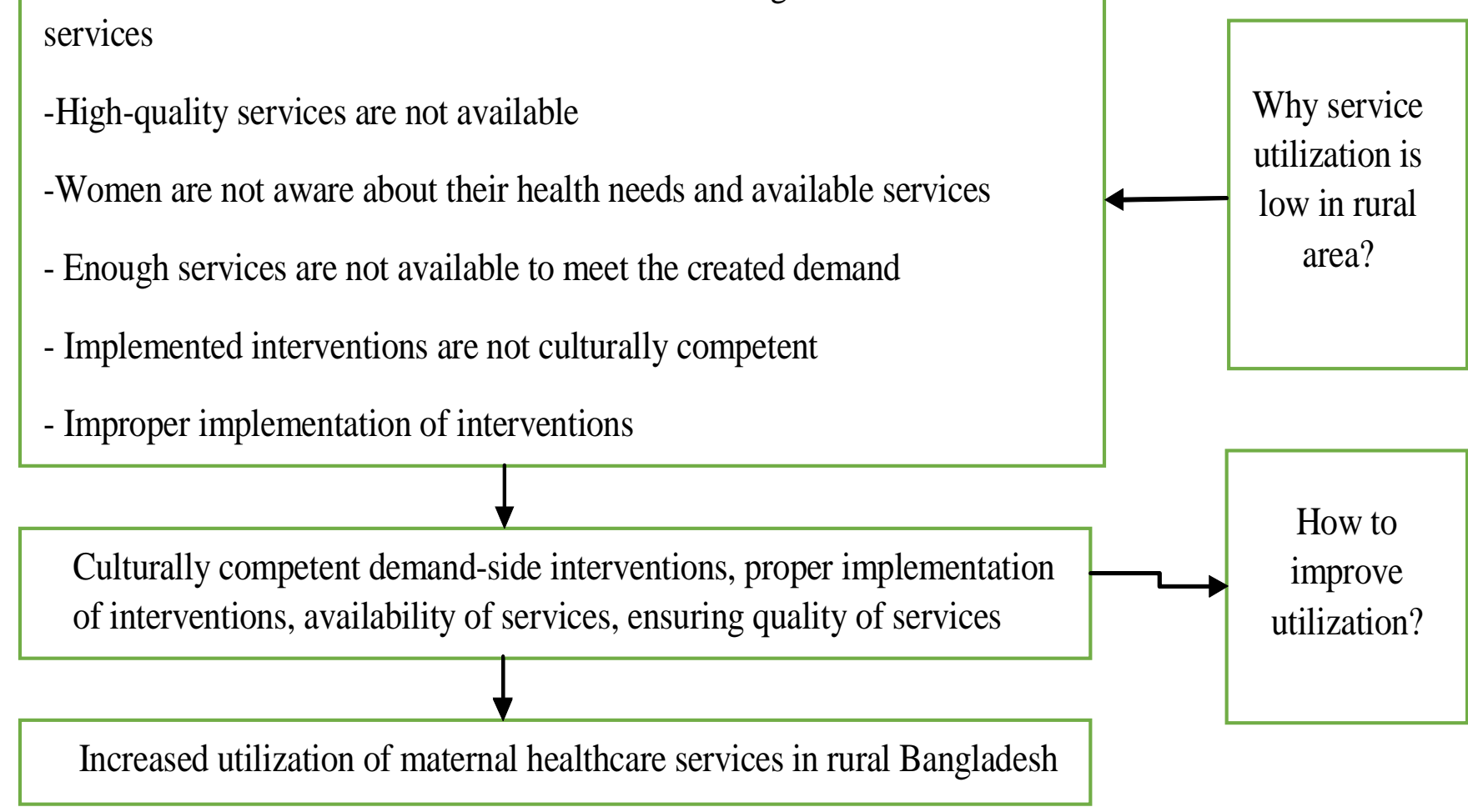

Figure-1: Step by step process to reach the hypothesis.

\section{Disparities according to level of wealth, education and area of residence:}

According to Pulok et al, maternal healthcare utilization has been persistently lower among poor, less educated and the people living in rural andremote areas in Bangladesh ${ }^{4}$. Household wealth status is significantly related to the disparities in maternal healthcare utilization. Multiple studies presented evidence on the association between wealth index and healthcare utilizaton. Pulok et al reported higher utilization of ANC and facility delivery among the richer households compared to the poorest ${ }^{4}$. Regarding CCs awareness and utilization, studies showed similar association. The study of Yaya et al revealed higher awareness about CCs and higher utilization of CCs among women having higher educational achievement and living in richer families ${ }^{8}$. 
Similar findings were reported in the study of Sarkar et al ${ }^{7}$. The both studies documented evidence on significant positive association of wealth status and level of education with CCs awareness and utilization. Ali et al revealed that higher educated adolescents and women were more likely to receive at least 4 or more ANC visits which is WHO recommened compared to the uneducated women in selected rural areas of Bangladesh ${ }^{9}$. Apart from women's education status, husbands' level of education is also considred as significant predictor causing disparities in healthcare utilization in rural Bangladesh ${ }^{4}$.

Mothers' area of residence also acts as a significant determinant of disparities regarding healthcare utilization. Studies reported significant diiferences in the findings of healthcare utilization between rural women and urban women. Pulok et al manifested in their study that, although coverage level of ANC and facility birth increased between the year of 2004 and 2011, utilization level was not reported as much improved as the coverage level${ }^{4}$. This study revealed high level of maternal health services utilization including of 2+ TT (Tetanus) injection, at least 4 ANC visits, skilled attendance at birth, and delivery at facilities among the urban women compared to rural ${ }^{4}$. But according to the study of Sarkar et al, women in urban areas were less aware about CCs compared to the women in rural area, and thereby utilization of CCs was evidenced less in urban area compared to rural area $^{7}$. Similarly, Yaya et al addressed in their study that women from poorer families were more aware of CCs and utilize CCs more compared to the women from richer families ${ }^{8}$. In Bangladesh, the trained healthcare providers are concentrated in major cities, whereas semiqualified or unqualified healthcare providers are more skewed to rural $\operatorname{areas}^{10}$. This situation contributes to lower quality of care in rural areas. In addition, more quality of care and lower waiting time are ensured in the private healthcare facilities compared to the public facilities in Bangladesh. Since, women in urban area are comparatively more educated and belong to comparatively richer households than rural areas, thereby they become comparatively more aware of their healthcare needs and tend to spend out of pocket to access private healthcare facilities. This situation implies that more level of wealth leads to more accessibility of high quality care.

Disparities according to administrative region and employment status: According to study findings, disparities in maternal healthcare utilization is also prominent across different administrative regions in Bangladesh. Pulok et al stated in their study that the uptake of 4+ ANC visits and ANC from a medically trained provider were low in Sylhet and Chattogram division of Bangladesh due to the fact that there are hilly areas in these two administrative division ${ }^{4}$.

Literature suggested, mothers' employment status has significant influence on disparities in healthcare utilization in Bangladesh ${ }^{4,11}$. Pervin et al specified in their study that female employment has significant association with Birth Preparedness and Complication Readiness (BP/CR) among rural women in Bangladesh ${ }^{11}$. Pulok et al documented in their study that microcredit membership of rural women has significant positive association with ANC service utilization in rural areas of Bangladesh ${ }^{4}$.

3. Effects of barriers to access maternal healthcare on its uptake in rural Bangladesh: There are various dimensions of barriers to access health care outlined in literature including financial barriers, cultural stigma, religious views, lack of healthcare awareness and transportation barrier.

Effect of financial barrier: Financial barrier is one of the most significant barriers influencing maternal healthcare utilization in developing countries including Bangladesh. Several studies manifested higher utilization of maternal healthcare services among the richer households compared to the poorer households ${ }^{7,89}$. Ali et al demonstrated in their study that adolescents and adult women from richest quintile showed higher tendency of ANC utilization and had more likelihood to receive ANC services compared to the poorest quintile 9 . On the other hand, in case of CCs utilization, the finding is completely opposite. Studies reported higher CCs utilization among the poorer and middle-class women compared to richer women ${ }^{7,8}$. Affordability and quality of care can explain this inconsistent finding. The CCs are public establishment and provide services for free. However, the quality of care provided in CCs is not ensured. Thereby women from richer households spend out of pocket to access high quality care from private facilities, and women from poorer households prefer the care which is affordable.

Effect of cultural stigma, religious views and lack of healthcare awareness: Cultural stigma and different religious views in utilizing facility-based healthcare pose significant barriers to access maternal healthcare services in rural Bangladesh. Besides, studies showed that cultural stigma attributes to lack of awareness and lack of knowledge about healthcare. Sarkar et al mentioned in their study that cultural and religious views might be the striking factors behind low level of health awareness and treatment seeking behavior in Bangladesh ${ }^{7}$. Bangladesh is a Muslim majority country where religious views in rural area permits limited mobility of women and prefers giving birth at home. Religious views do not promote school- based education of adolescent girls, which eventually leads to low level of health awareness among the rural women. Pulok et al indicated low level of literacy and religious conservatism in the regions showing low level of maternal health care service utilizations in Bangladesh4. Apart from posing as barrier to access maternal healthcare services, cultural stigma acts as a major challenge for developing nations in accessing demand-side interventions implemented to improve utilization. Research demonstrated that cultural context is inadequately considered in the design and implementation of demand side interventions and strategies to improve access to maternal healthcare ${ }^{3,12,13}$.

Review from literatures suggests that there are three fundamental cultural factors influence the decision-making environment for the 
woman. These influences are: Gendered decision-making norms, multigenerational dialogue and appropriate communication ${ }^{12}$. Gendered decision-making norms creates unequal power in the decision-making process within households which further limits women's power to negotiate with her partner, thereby increasing fertility rates, unwanted pregnancy, and negatively affect maternal health ${ }^{12}$. Older generation women don't feel comfortable to share motherhood experiences with their daughters due to cultural sensitivity ${ }^{12}$. Thereby, cultural stigma creates wider gaps in the knowledge transmission across multiple generations, later results in young girls embarking on motherhood with little knowledge on major life-threatening danger signs associated with pregnancy and delivery ${ }^{12}$. Besides, due to social stigma and cultural sensitivity, there exists a lack of appropriate communication between pregnant women and health care providers causing low utilization of accessible antenatal care ${ }^{12}$.

Effect of lack of exposure to mass-media: Lack of exposure to mass media leads to a lack of information about health awareness which also acts as a barrier to access healthcare. Moinuddin et al alluded to an example of a study conducted in Uganda where a significant positive association was found between mass media exposure and birth preparedness ${ }^{14}$. The authors also mentioned that the women in Bangladesh who read newspaper were more likely to plan for safe delivery and suggested some level of literacy ${ }^{14}$. Several other studies recommended a high level of maternal health service utilization among the rural women in Bangladesh who were exposed to mass media ${ }^{4,9,11}$. Ali et al documented in their study that adolescent and adult women who were exposed to mass media were 1.62 times more likely to receive ANC from a facility compared to those who were not exposed to mass media?

\section{Effect of transportation barrier:}

Transportation issue is a major barrier to access healthcare in rural and remote areas of Bangladesh, especially in hard to reach areas. Pervin et al found in their study that majority of women participated in the study planned to have birth at home, and this finding was more prominent in the areas in Bangladesh where access to healthcare facilities considered as challenging ${ }^{11}$. Also, a similar finding was demonstrated in the study conducted by Moinuddin et al where the authors revealed that due to the affordability issue and to facilitate transport to facilities during emergency, 'saving money' was the most observed component as birth preparedness/ complication readiness (BP/CR) for child birth and managing complications among the rural women in hard-to-reach areas of Bangladesh ${ }^{14}$.

Impact of demand-side interventions on healthcare utilization in developing countries: Demand-side interventions in maternal health care are defined as interventions implemented to increase utilization of maternal health services either through financial incentives (cash transfers, vouchers) or through the provision of information by participatory women's groups or other community-based education efforts ${ }^{3}$. In general, 'demand- side intervention' means the interventions which are developed and implemented to change the healthcare seeking behavior of recipients ${ }^{15}$. These interventions are designed to increase uptake through financial incentives that reduce the cost of accessing services or through community mobilization efforts to improve knowledge about available services and address cultural attitudes which may prevent uptake of potentially life-saving services ${ }^{3}$. Several studies determined that implementation of demand-side interventions contributed to improve uptake of health services and improved health outcomes. There are numbers of demand-side interventions mentioned in available researches including community mobilization activities such as participatory women's groups, training of community facilitators, enhanced patient transfer and community level health promotion; financial incentives such as voucher schemes and conditional cash transfers (CCT) $)^{3,12}$. Elmusharaf et al demonstrated effectively of voucher system as a form of demand-side funding in many developing country settings to improve access to pre-defined services in case of targeted hard-to-reach populations ${ }^{12}$. As an illustration, the authors included a brief description of a quasiexperimental study conducted in Uganda which showed a rapid increase in the utilization of maternal care through voucher system ${ }^{12}$. However, improving utilization always does not translate into improved health outcomes. Hurst et al conducted a literature review to understand the current evidence of demand-side interventions' impact on improving utilization and outcomes for mothers and newborns which includes a total of 16 articles and out of 16 articles only 4 articles reported both increased utilization and decreased mortality through implementing community mobilization activities and financial incentives ${ }^{3}$.

Research documented that demand-side interventions may not be enough to improve utilization if issues such as the wider cultural context, inequality, transportation system limitations, cost effectiveness, health system strengthening and sustainability are not considered and integrated ${ }^{16,17}$. Other factors which were considered in literature to affect changes in health outcomes areintensity and coverage of interventions ${ }^{3}$. These factors explain the effectiveness of community mobilization activities such as participatory learning model. According to the study of Hurst et al participatory learning model worked as a cost-effective strategy in Bangladesh to improve maternal and neonatal survival when at least a third of pregnant women participated in the intervention ${ }^{3}$. Ari et al recommended several reasons behind not registering for the CCT program in North Central Nigeria including- spousal and community disapproval, long waiting times, lack of availability of free drugs, long distance to health facilities, the quality of care received at the facilities and fears of poor treatment by the health workers ${ }^{13}$. Alongside implementing any demand- side intervention, assessing the effectiveness or efficacy of the intervention would be challenging considering few factors including lack of knowledge about the context, lack of system-wide integration and 
lack of quality evidence on outcome ${ }^{12}$. Tobe et al developed a study protocol to improve maternal and child health care in rural Bangladesh where the authors incorporated the $\mathrm{MCH}$ handbook with mobile short messages and audio reminders in local languages for suitability to illiterate, to ensure data integrity2. In the study, this culturally sensitive innovative intervention packagethe combination of mobile phone network and $\mathrm{MCH}$ handbook is expected to maximize advantages in terms of knowledge dissemination, promotion of communication, preservation of home-based health records, and facilitation of continuum of perinatal care ${ }^{2}$.

Elmusharaf et al described the effectiveness of provision of a few culturally sensitive strategies including emergency transport fund; immediate alternative forms of transportation and maternity waiting homes. Immediate forms of transportation such as motorized transport (motorcycles, pick-up trucks, tractors and motorboats) and non- motorized transport (bicycles, animal drawn carts and canoes) facilitate geographical accessibility to improve utilization of maternal care ${ }^{18}$. Reviews of literature suggested that motorized transports that consider cultural concerns are more likely to be an acceptable and effective choice for pregnant women during emergencies ${ }^{18,19}$. In rural areas of Bangladesh, motorized transports such as tractors, motor boats are likely to be an acceptable form of transportation, since people use it in their daily life for agricultural and fishing purposes, also for social connection. Maternity waiting homes are residential facilities within easy reach of emergency obstetric care (EmOC) that provide a place to stay for high-risk pregnant women and for those who are living far away during the final weeks of their pregnancy ${ }^{12}$. Elmusharaf et al also alluded to an example of conditional incentive program implemented in Nepal for all pregnant women that encourages institutional delivery; later this intervention faced severe constraints such as bureaucratic delays, lack of planning and weak and inadequate health services ${ }^{12}$. This example signifies the importance of ensuring government support and strategic planning before implementation of any demand-side intervention in lower- and middle-income countries.

\section{Discussion}

According to evidence reported in literature, Bangladesh made noteworthy progress across different health indicators, especially maternal and newborn health-related indicators. However, a deeper look into key indicators of Bangladesh revealed significant differences in rich-poor, urban-rural and other socio-demographic factors in terms of access to and utilization of maternal care. Findings from studies reported significant healthcare disparities among different geographical divisions as well. It was perhaps because there are hilly areas and hard to reach areas in several divisions in Bangladesh where service coverage is consistently ${ } \mathrm{w}^{4}$. Additionally, findings documented that women exposed to mass media were more likely to use maternal health care services ${ }^{4,9}$. Explanation for this finding is possibly the increased dissemination of health education message through popular mass media ${ }^{4,9}$. Studies recommended, along with mass media exposure, demand side interventions and strategies aiming to increase appropriate care-seeking behavior are critical to improve utilization. Having said that several factors should be taken into account before implementing any demand- side interventions, such as degree of urbanization, population and facility level targeted, existing health infrastructure, differences in geographical accessibility of services, community perceptions of the maternal health services and quality of existing facility-based care $^{3}$. However, demand-side interventions alone cannot ensure utilization of healthcare, since study regarding conditional cash transfer documented that the poor quality of healthcare services provided at the PHC (Primary Health Center) was one of the perceptions the non-beneficiaries cited as a reason for not registering for the CCT services provided ${ }^{13}$. According to Ari et al improving the quality of care will lead to better patient satisfaction and consequently improve health seeking behavior and utilization of government-provided healthcare services like the CCT programs ${ }^{13}$. Since patient perception of quality is critical in the utilization of healthcare services, the availability of adequate number of facilities and availability of health workforce needs to be weighed before a country decides to roll out a CCT program. Studies also revealed that government programs tailored to women empowerment such as women microcredit membership increases women's decision-making autonomy which further leads to increased utilization of healthcare ${ }^{4}$.

\section{Conclusion and Recommendations}

In conclusion, the analysis above presented evidence on factors that contribute to inequities in terms of availability and utilization of maternal healthcare in rural areas of Bangladesh and on demand-side interventions implemented to overcome the inequity in developing countries, including Bangladesh. The analysis above also highlighted the importance of considering existing barriers perceived by rural women and other cultural contexts before adopting different approaches tailored to enhanced utilization. The government should prioritize strategies that strengthen the healthcare delivery system, build on healthcare services that are available, accessible and acceptable to the population across the nation. Concurrently, special attention should be given to formative evaluation to ensure that the program or policy to be implemented is feasible, appropriate, and acceptable to the targeted communities, resulting in the desired impact on maternal mortality.

\section{References}

1. Brolan CE, Hill PS. Universal Health Coverage's evolving location in the post-2015 development agenda: Key informant perspectives within multilateral and related agencies during the first phase of post-2015 negotiations. Health Policy and Planning 2016; 31(4):514-26.

2. Tobe RG, Haque SE, Ikegami $K$ et al. Mobile-health tool to improve maternal and neonatal health care in bangladesh: A cluster randomized controlled trial. BMC Pregnancy Childbirth 2018; 18:102. 
3. Hurst TE, Semrau K, Patna M et al. Demand-side interventions for maternal care: Evidence of more use, not better outcomes. BMC Pregnancy Childbirth 2015; 15:297.

4. Pulok MH, Sabah MNS, Uddin J et al. Progress in the utilization of antenatal and delivery care services in bangladesh: Where does the equity gap lie? BMC Pregnancy Childbirth 2016; 16:1-16.

5. WHO. Success Factors for Women's and Children's Health: Bangladesh. Available at https://www.who.int/pmnch/knowledge/ publications/ bangladesh.pdf

6. WHO. Success Factors for Women's and Children's Health: Bangladesh, 2015. https://www.who.int/pmnch/knowledge/ publications/ bangladesh.pdf

7. Sarkar MAB, Harun-Or-Rashid M, Reyer JA et al. Associations of socioeconomic determinants with community clinic awareness and visitation among women: Evidence from bangladesh demographic and health survey-2011. BMC Res Notes 2015; 8:1-8.

8. Yaya S, Bishwajit G, Ekholuenetale M et al. Awareness and utilization of community clinic services among women in rural areas in Bangladesh: A cross-sectional study. PLOS ONE 2017; 12(10):e0187303.

9. Ali N, Sultana M, Sheikh N et al. Predictors of Optimal Antenatal Care Service Utilization Among Adolescents and Adult Women in Bangladesh. Health services research and managerial epidemiology 2018; 5:1-8.

10. Joarder T, Rawal LB, Ahmed SM et al. Retaining Doctors in Rural Bangladesh: A Policy Analysis. International journal of health policy and management 2018; 7(9):847-58.

11. Pervin J, Nu UT, Rahman AMQ et al. Level and determinants of birth preparedness and complication readiness among pregnant women: $A$ cross sectional study in a rural area in Bangladesh. PLOS ONE 2018; 13(12): e0209076.
12. Elmusharaf K, Byrne E, O'Donovan D. Strategies to increase demand for maternal health services in resource-limited settings: Challenges to be addressed . BMC Public Health 2015; 15:870.

13. Baba-Ari F, Eboreime EA and Hossain M. Conditional Cash Transfers for Maternal Health Interventions: Factors Influencing Uptake in North-Central Nigeria. International journal of health policy and management 2018; 7(10):934-42.

14. Moinuddin M, Christou A, Hoque DME et al. Birth preparedness and complication readiness (BPCR) among pregnant women in hard-to-reach areas inBangladesh. PLoS ONE 2017; 12(12): e0189365.

15. Standing $\mathrm{H}$. Understanding the 'demand side' in service delivery: definitions, frameworks and tools from the health sector. DFID 2004:1-42. http://www.heart-resources.org/wp-content/uploads/2012/10/Understandi ng-the-demand-side-in-service- delivery.pdf

16. Ensor T. Consumer-led demand side financing in health and education and its relevance for low and middle income countries. Int $\mathrm{J}$ Health Plann Mgmt 2004; 19: 267-285.

17. Pariyo G, Mayora C, Okui O et al. Exploring new health markets: Experiences from informal providers of transport for maternal health services in Eastern Uganda. BMC International Health and Human Rights 2011; 11(Suppl 1):1-11.

18. Holmes W and Kennedy E. Reaching emergency obstetric care: Overcoming the 'second delay'. Burnet Institute on behalf of Compass, the Women's and Children's Health Knowledge Hub. Melbourne, Australia; 2010.

19. Krasovec K. Auxiliary technologies related to transport and communication for obstetric emergencies. International Journal of Gynecology and Obstetrics 2004; 85(1):S14-S23. 